Background: Wildfires occur where all elements of the fire triangle exist, these are an ignition source, combustible material and oxygen. During December 2010 Israel's Carmel forest experienced an extensive forest fire with 17,000 people evacuated from their homes and 44 dead, the fire did not extend out of the region where it started. During November 2016, the weather conditions were hot and dry and fires occurred in multiple regions in Israel, some not close to each other. The police investigations are yet to have final conclusions but preliminary reports show that 40/90 fires are due to arson.

Methods: Magen David Adom (MDA), the Israeli national EMS organization compared the difference between arson terror and wildfires from EMS perspective.

Results: Fires occurred in different regions of Israel, more than 1,500 apartments were consumed and 75,000 people evacuated. Two nursing homes were evacuated with more than 80 patients transported, including an ICU ward with 22 mechanically ventilated patients. More than 125 casualties were evacuated with smoke inhalation injuries. MDA resources utilized include 845 ambulances, 61 medicycles, 99 mobile intensive care units (MICUs), 2 mass casualty incident (MCI) vehicles, an advanced command \& control vehicle and emergency backup ambulances. Conclusion: Arson terror is characterized by multiple fires in different regions. The Magen David Adom response to extensive incidents is based on shift, on-call response and recruiting personnel and vehicles from nearby regions. Mobilization of EMS resources is complicated because of fires blocking roads, the need to simultaneously respond in multiple regions while holding preparedness in others because of the uncertainty factor about the locations of next arsons. Also, although they had been called, not all personnel can report to duty because their homes or families are affected.

Prehosp Disaster Med 2017;32(Suppl. 1):s166-s167

doi:10.1017/S1049023X17004496

\section{Simulating Multi Casualty Incidents to Improve Preparedness of Potential Incident Commanders for Real Events

Avi Parush ${ }^{1}$, Eli Jaffe ${ }^{2}$, Tal Solomon ${ }^{1}$, Roman Sonkin ${ }^{2}$ \\ 1. Industrial Engineering And Management, Israel Institute of Technology, Haifa/Israel \\ 2. Community Outreach, Magen David Adom in Israel, Tel Aviv Jaffo/Israel}

Study/Objective: To study the influence of Mass Casualty Incident (MCI) simulator on the confidence and decisionmaking of potential incident commanders.

Background: Israel has been dealing with multi casualty incidents since before it was founded. Magen David Adom (MDA) as the Israeli national EMS organization, has gained extensive MCI experience. MDA personnel are trained in the concepts and algorithms of MCIs and incident commander training is mandatory for EMT's and Paramedics. A simulator was developed with cooperation of "Technicon" institute to simulate the high load on the thought processes while making decisions. The participant is briefed at the beginning of the scenario, then he must follow the initial MCI algorithm and start making decisions to distribute forces, triage and patient treatment.
The simulator does not allow delegation of medical, parking and transportation responsibilities to deputy commanders.

Methods: The simulator was played by MDA personnel in a classroom mode. Participants were given 10 minutes per scenario, each participant played 3-5 scenarios after which, the participants had to fill out a survey about the scenario, and a concluding survey after the completion. The logs were retrieved and compared to the surveys to analyze change in confidence level as incident commander, action times and durations, prioritization skills and overall survival of casualties.

Results: With the completion of each scenario the participants reported a rise in their confidence level as incident commander, shorter response times for requests for additional resources, and shorter times for initial triage and patient treatment, and transportation to the hospitals.

Conclusion: The simulator engages participants to make quick and appropriate decisions while in state of stress. The main goal is to save as many lives as possible by conducting good initial triage and lifesaving treatment, transporting as many patients as possible - as quickly as possible and with the most appropriate medical personnel. The simulator was found to increase confidence, decision making and prioritizing among incident commanders.

Prehosp Disaster Med 2017;32(Suppl. 1):s167

doi:10.1017/S1049023X17004502

\section{Developing an Educational Intervention to Train Prehospital Responders in High Consequence Emerging Infectious Diseases \\ J Lee Jenkins, Richard Bissell \\ Emergency Health Services, Univ of Maryland Baltimore County, Baltimore/MD/United States of America}

Study/Objective: The goal of this session is to provide participants with an overview of efforts currently underway, to develop an interactive online curriculum to provide an awareness level, as well as, just-in-time training for issues surrounding response to high consequence emerging infectious diseases. Background: From the global threat of Ebola Viral Disease, to outbreaks of novel influenza, through localized outbreaks of multidrug resistant tuberculous, the prehospital disaster and emergency medical community must continue to maintain a constant awareness of operational and clinical concerns associated with high consequence emerging infectious diseases. Such vigilance starts with syndromic recognition, and quickly transcends to include operational issues, clinical interventions, public health integration.

Methods: The University of Maryland, Baltimore County (Maryland, USA), Department of Emergency Health Services has partnered with the Maryland State Department of Health and the Centers for Disease Control and Prevention (USA), to develop an online educational curriculum. The curriculum is hybrid in design and includes awareness level training, as well as, just in time "medical minutes" for providers to review in near, real time. Once deployed, the content will be accessible via computer, tablet and smartphones. The curriculum is validated by subject matter experts and field providers for content and usability. 
Results: Scheduled for deployment in mid 2017, this curriculum will be accessible to over 50,000 prehospital, hospital and clinic personnel throughout Maryland and the National Capital Region of the United States, as well as internationally through the web interface. Curriculum exists of twelve modules of didactic and live video-taped demonstrations.

Conclusion: Online education has been established as a wellvalidated means of content delivery, and offers an ideal means of content distribution to prehospital personnel. The development of an online educational intervention to educate prehospital personnel in critical issues surrounding high consequence emerging infectious diseases, can help ensure better patient care and prehospital EMS system readiness.

Prehosp Disaster Med 2017;32(Suppl. 1):s167-s168

doi:10.1017/S1049023X17004514

Three-Wheeler Driver Training on Prehospital Emergency Care Service Provision in Anuradhapura Sri Lanka Ayanthi S. Karunarathne ${ }^{1}$, Samarage ${ }^{2}$, Kumarapeli ${ }^{3}$

1. Administration, Teaching Hospital Anuradhapura, Anuradhapura/ Sri Lanka

2. Institute for Health Policy, Colombo/Sri Lanka

3. Ministry of Health Colombo Sri lanka, Colombo/Sri Lanka

Study/Objective: To improve the capacity of Three-Wheeler Drivers (TWDs) on prehospital emergency care provision and patient transport in the City of Anuradhapura.

Background: Since the Tsunami of 2004, the most destructive natural disaster in the country Government of Sri Lanka, together with a number of private organizations, attempted to establish prehospital care service provisions; however they failed to establish such to date. World Health Organization (WHO) recognizes, the development of a lay person first responder program, as the initiation towards establishing proper EMS in resource poor settings. Anuradhapura is geographically the largest district situated $220 \mathrm{Km}$, (137 mi.) away from the Capital.

Methods: A descriptive study was carried out over a period of two weeks in the Teaching Hospital of Anuradhapura (THA), the only tertiary care center in the district, to identify the contributions made by the TWDs on emergency patient transport. A group of TWD $(\mathrm{N}=37)$ was trained on first aid, and some components of BLS and safe patient transport. A training module was developed with the aid of consultant anesthetists, surgeons and triage nurses working in THA. Pre and post assessments were compared to assess the effectiveness of the training program. Results: Nearly three quarters of patients admitted to the emergency medical and surgical units were transported in TWS $(74,7 \%, \mathrm{n}=454)$. Cardiovascular incidents including MI $(14.9 \% \mathrm{n}=68)$, snakebite and poisoning $(3.3 \%, \mathrm{n}=15)$ were the most common medical emergencies; while Trauma including RTA was the most common surgical emergency $(44.9 \%, 204)$. Participants for the training program had an average of 13 years (SD 5.4) experience as a TWD, and has handled 12 emergency patients a year $(\mathrm{SD}=7.95)$. Nevertheless, none of them has had a previous exposure to training on EMS; Paired $t$ test showed significant improvement on the post training assessment $(t=16.954,95 \%$ CI6.47tp 8.23, $\mathrm{p}<0.00)$.
Conclusion: Considering the pattern of emergency patient handling in the area, TWDs could be the best layperson group to train on EMS. Training module should be designed in a way to address the most common emergency conditions.

Prehosp Disaster Med 2017;32(Suppl. 1):s168

doi:10.1017/S1049023X17004526

\section{Death in an Ambulance in Rural Haiti: Proper Care of the Recently Deceased \\ Benjamin Nicholson \\ Department of Emergency Medicine, Boston Medical Center, Boston/MA/United States of America}

Study/Objective: To describe the issues encountered during the profession of emergency care to a severely injured patient, who ultimately died during transport.

Background: Severely injured patients can die during ambulance transport to a hospital. The appropriate care and disposition of the deceased varies greatly depending on location, and carries significant implications.

Methods: The case was discussed with providers involved in the care of the patient, and the local Haitian staff who assisted in the ultimate disposition of the body.

Results: A severely injured man involved in a motor vehicle collision in rural Haiti was transported by ambulance to the nearest trauma hospital. No identification of the patient or his next of kin was possible. En route, the patient became pulseless despite active resuscitation. On arrival, a non-national physician entered the ambulance and declared the patient deceased, prior to accepting patient care or allowing the patient to leave the ambulance. He then refused to accept the deceased, stating the hospital lacked storage facilities and the resources to identify the next of kin. Consultation with the Haitian director of the clinic where the ambulance was based, led to the decision to return the deceased to the clinic and await the police. Following a police investigation, the deceased was transferred to the national hospital morgue.

Conclusion: Proper care of the deceased is a highly sensitive cultural matter. In this case, the clinic director's advice for future incidents was to await next of kin before transport in all cases. In regions where prehospital care is uncommon, cultural beliefs and legal statutes may not take into account the ramification of delayed care for the critically injured. Developing an understanding of local, legal and culturally acceptable means of properly caring for severely injured patients who die, is paramount to any international medical operation.

Prehosp Disaster Med 2017;32(Suppl. 1):s168

doi:10.1017/S1049023X17004538

\section{Does Rotor Wing Evacuation Shorten Total Prehospital}

Time? Analysis of Data from Southern Israel

Dagan Schwartz ${ }^{1}$, Zeev Avraham ${ }^{2}$

1. Emergency Medicine, Rabin Medical Center, Petach-Tikva/Israel

2. Emergency Medicine, Ben-Gurion University of the Negev, Beer-Sheva/Israel

Study/Objective: To evaluate the effect of rotary wing evacuation by the Israeli air force medevac unit on prehospital time in the south of Israel. 